\title{
DIRECCIÓN CORAL Y TÉCNICA VOCAL, ¿UN DIÁLOGO POSIBLE? REFLEXIONES METODOLÓGICAS PARA UN TRABAJO VOCAL EFICIENTE.
}

\author{
Choral Conducting and Vocal Technique: Is dialogue possible? Metodological \\ considerations for an efficient vocal work.
}

\begin{abstract}
Sixty years ago, the operation of the voice as an instrument governed by acoustic rules and belonging to the human body and its physiological mechanisms was a mystery. Nowadays, thanks to science research, it is possible (and unavoidable) to introduce the voice from a scientifically based perspective. Choral practice is vocal practice, so that it has become essential for the choral conductor to be acquainted with new knowledge regarding Vocal Technique and Vocal Pedagogy. There are choir conductors and voice professionals who don't know how the vocal instrument comprises in detail, what are their most important features; what are the nervous, sensorial and motor processes involved in the phonation process, and how to perceive the difficulties and kinesthetic sensations of their singers. This implies a real obstacle for the professional development of the activity. The challenge that choir directors are facing in the XXI century is linked to the possibility of apprehending and applying available knowledge in pursuit of constant improvement and planned perfectioning of vocal sound. This article proposes a path through the most important concepts of Contemporary Vocal Pedagogy (as diagnostic, prescription and vocal training) and delineates the crosslinks of interest for the choir conductors of this new scientific era.
\end{abstract}




\section{Resumen}

Hace 60 años el funcionamiento de la voz en tanto instrumento regido por las leyes acústicas e inscripto en el cuerpo humano, y por lo tanto, gobernado por los mecanismos fisiológicos, era un misterio. Hoy en día, gracias a los avances de la ciencia, es posible (y resulta inevitable) presentar la voz desde una perspectiva sólidamente fundamentada. La práctica coral es práctica vocal, y por lo tanto, para el director coral resulta fundamental estar familiarizado con los nuevos conocimientos disponibles en el área de la Técnica Vocal y la Pedagogía del Canto. Existen directores corales y profesionales de la voz que no conocen en detalle cómo está compuesto el instrumento vocal, cuáles son sus características más importantes, cuáles son los procesos nerviosos, sensoriales y motrices implicados en el proceso fonatorio, y que no son capaces de observar y percibir las dificultades y sensaciones que sus coreutas poseen. Esta situación se presenta sin dudas como un obstáculo para el desarrollo profesional de la actividad que no encuentra, en la mayoría de los casos, una solución fundamentada y efectiva. El desafío que enfrentan los directores corales en el siglo XXI se encuentra vinculado a la posibilidad de aprehender e instrumentalizar el conocimiento disponible en pos del perfeccionamiento constante y la mejora planificada del sonido vocal. Este artículo propone un recorrido por los conceptos más importantes de la Pedagogía Vocal Contemporánea (tales como los de diagnóstico, prescripción y entrenamiento vocal) y delinea los entrecruzamientos de interés para los directores corales de esta nueva era científica.

Keywords: Vocal Technique - Singing - Contemporary Vocal Pedagogy - Diagnostic.

Palabras Clave: dirección coral - técnica vocal - pedagogía vocal - diagnóstico - entrenamiento.

Data de submissão: Dezembro de 2011 | Data de publicação: Junho de 2012.

NICOLÁS ALESSANDRONI - Facultad de Bellas Artes - Laboratorio para el Estudio de la Experiencia Musical (LEEM) Universidad Nacional de La Plata (U.N.L.P). Correo electrónico: nicoalessandroni@hotmail.com

ESTEBAN ETCHEVERRY - Facultad de Bellas Artes - Universidad Nacional de La Plata (U.N.L.P). Correo electrónico:tebane@ hotmail.com 


\section{Introducción: el instrumento vocal en el siglo XXI}

Hace 60 años el funcionamiento de la voz en tanto instrumento regido por las leyes acústicas e inscripto en el cuerpo humano, y por lo tanto, gobernado por los mecanismos fisiológicos, era un misterio. La voz humana se configuraba como un instrumento totalmente invisible. Hoy en día, gracias a los avances de la ciencia, es posible (y resulta inevitable) presentar la voz desde una perspectiva sólidamente fundamentada. Para ser más precisos, a continuación analizaremos estas cuestiones.

El instrumento vocal, tal como el resto de los instrumentos, está compuesto por 3 partes: una fuente de energía, un vibrador y un resonador (VENNARD, 1949; ROTHENBERG, 1981, \& SUNDBERG, 1987). La voz posee su fuente de energía en todo el cuerpo del cantante, pero la energía principal la aporta específicamente el sistema respiratorio a través de la columna de aire que se genera por debajo de la glotis (espacio entre las cuerdas vocales). El sonido vocal se produce por la vibración de las cuerdas vocales (vibrador) que responden a impulsos nerviosos durante el pasaje de aire. Para ser más precisos describiremos cómo es un ciclo fonatorio: en principio la glotis se abre y los pliegues vocales se separan para permitir la entrada de aire en la inspiración. Ahora bien, luego de la inspiración, la glotis posee tendencia al cierre por acción de los músculos interaritenoideos y cricotiroideos laterales. Es en este instante en el que se activan ciertos mecanismos motores sobre los pliegues vocales a partir de la imagen mental de una palabra o de un sonido (MAULÉON, 2008). Los músculos tiroaritenoideos (pliegues vocales) se contraen posicionándose de acuerdo a la frecuencia a emitir. Al posicionarse la glotis de este modo, los pliegues vocales quedan aproximados en forma suficiente como para que la ondulación de su mucosa provoque sucesivas interrupciones en la columna de aire ascendente (también conocida como presión subglótica). La cantidad de ondulaciones de la mucosa de los pliegues vocales en función de una unidad de tiempo determinarán igual cantidad de interrupciones en la columna de aire ascendente, y esto dará por resultado la frecuencia del sonido emitido a nivel laríngeo, el cual se conoce con el nombre técnico de frecuencia fundamental. Finalmente, el sonido es enriquecido y amplificado en el tracto 
vocal que actúa como resonador y comprende la cavidad oral, se extiende a lo largo de la faringe, el vestíbulo laríngeo y finaliza en las cuerdas vocales (RABINE, 1999).

Una de las diferencias de la voz con el resto de los instrumentos es la capacidad de articular fonemas, característica que le aporta infinitas posibilidades respecto el resto de los instrumentos. Por otro lado y como hemos dicho, el instrumento vocal se encuentra inscripto en el cuerpo del individuo, por lo cual la salud psíquica y física constituyen aspectos de suma importancia para el cantante. Paralelamente, en lo que respecta al entrenamiento, es necesaria la adquisición de nuevas habilidades y la reprogramación de esquemas asociados previamente a otras funciones vitales primarias (como la respiración o la deglución), para que al momento de ejecutar el instrumento, el cantante pueda optimizar al máximo el dominio de las estructuras implicadas en el canto (MAULÉON, 2001).

Por último, hemos de decir que el hecho de que el cantante sea a su vez instrumento e instrumentista determina que cada instrumento vocal sea diferente: cada persona posee características anatómicas y psíquicas particulares que deben ser atendidas. Esta última afirmación resulta clave para re-pensar la pedagogía vocal, ya que muchos de los modelos asociados a la enseñanza del canto se han apoyado históricamente (y se siguen apoyando) en la imitación. En el próximo apartado analizaremos esta cuestión.

\title{
Sobre el concepto de Entrenamiento Vocal
}

\author{
Así como ustedes piensan, así enseñan. Y de acuerdo \\ a como piensan, así cantan. (RABINE, 2002)
}

De la mano del advenimiento de la Pedagogía Vocal Contemporánea (1950 a la fecha) y de su confluencia con las nuevas tecnologías emergió un marco teórico eficaz para remplazar a la enseñanza por imitación. Por primera vez se pudo comprender la estructura anatómica, fisiológica y funcional del instrumento vocal. La enseñanza por imitación quedó en el olvido para dar paso a un concepto mucho más preciso: el de entrenamiento vocal (MILLER, 1986; RABINE, 2002). El análisis de las condiciones evolutivas del instrumento 
y de la función primaria de las estructuras fisiológicas que se ensamblan al cantar permitió comprender que la formación del cantante no se da de modo "natural", sino que debe entenderse como el entrenamiento de un esquema corporal-vocal diferente del que utilizamos para el habla, en el cual las estructuras intervinientes en la producción del sonido se ensamblan según los patrones más eficientes para cantar o, lo que es lo mismo, alcanzan el mayor grado posible de diferenciación funcional. Aún más, los teóricos contemporáneos advierten que durante la producción vocal tienen lugar ciertos procesos no conscientes (como por ejemplo, la actividad diafragmática o de ciertos músculos laríngeos) que deben ser reconfigurados de modo indirecto (actuando sobre otras estructuras que las afectan secundariamente).

Según Mark Johnson (1987), un esquema está constituido por patrones corporeizados de experiencia significativa organizada como estructuras de movimientos corporales e interacciones perceptuales; por lo tanto, para lograr construir el esquema corporal-vocal (propio del entrenamiento de la voz humana), será necesario contar con ciertos dispositivos pedagógicos que permitan organizar la experiencia sensorial de manera efectiva para el canto: estamos hablando de los ejercicios.

Un ejercicio es una herramienta que puede ser descripta, que requiere una actividad cuyo propósito es de aprendizaje y que cuando es repetida en el tiempo, tiene un efecto de entrenamiento (RABINE, 2002) .Así aplicado, un ejercicio tiene influencia sobre la conducta del cantante al modificar sus esquemas físicos y psíquicos, es eminentemente comunicativo en sí mismo y apoya también la comunicación hacia adentro. Durante la realización de un ejercicio, el alumno tiene la oportunidad de comparar su desempeño vocal previo con las nuevas sensaciones y resultados acústicos, de vivenciar y confrontar sus sensaciones fisio-acústicas momentáneas con su concepto mental y su intención comunicativa. Como resultado, el individuo encontrará nuevas propiocepciones que deberán ser seleccionadas, ordenadas y, eventualmente, nombradas. Los ejercicios, por lo tanto, permiten conceptualizar la práctica vocal para luego instrumentalizarla.

Se desprende de lo anterior que toda práctica profesional de enseñanza del canto debe contemplar un determinado tiempo de trabajo que permita el desarrollo de una estrategia didáctica conformada por secuencias de ejercicios direccionadas hacia un fin 
particular. Según cuál sea el caso, el maestro de canto o director coral deberá encontrar un marco adecuado para trabajar teniendo en cuenta los objetivos mediatos e inmediatos a cumplir (PARUSSEL, 1999).

De esta manera, será posible planificar un entrenamiento vocal de larga duración cuyos objetivos principales contemplen la enseñanza del canto desde el equilibrio de la función vocal (sistema postural y de movimiento, respiración, emisión, resonancia y articulación), la maduración de nuevos conceptos mentales, el reconocimiento de la voz por parte del cantante, y el desarrollo de un modo de producción vocal adecuado y eficiente para la comunicación artística. Por otra parte, cuando un cantante ha atravesado un proceso de entrenamiento, resulta conveniente el diseño de un precalentamiento vocal (HEIZMANN, 2003) cuyos objetivos principales serán la activación de la respiración, la evocación rápida de los patrones de coordinaciones corporales previamente ejercitados (incluida la postura general), el abordaje y resolución de las dificultades que presenten las obras a ejecutar, y la vinculación comunicacional y emocional con el repertorio previa performance pública.

Como veremos más adelante, la masterización de los recursos pedagógicos y metodológicos de la enseñanza del canto, le permitirá al director coral delinear estrategias didácticas más adecuadas y eficientes para impulsar el desarrollo de un sonido coral funcionalmente más balanceado.

\section{La vinculación entre la técnica vocal y el director coral}

El profesor de arte que indica a su alumno que "piense en violeta", o que solicita que "salgan tonos flotantes de una chimenea en la cabeza", ya no es tomado en serio (MILLER, 1996).

Para comenzar este apartado creemos importante que cada lector se realice los siguientes cuestionamientos: ¿Cuántos directores corales son realmente conscientes del poder que tienen para mejorar o deteriorar el instrumento vocal de sus coreutas y de la 
responsabilidad que esto conlleva? ¿Cuántos directores consideran la individualidad y las particularidades de cada instrumento vocal que poseen en sus coros? ¿Cuántos de ellos repiten ejercitaciones estandarizadas, arquetipizadas o modelos facilistas que carecen de prescripción y no han sido generados a partir del diagnóstico de las dificultades vocales de sus coreutas?

Podemos afirmar que todos los directores desearían estar a cargo de un grupo coral que suene bien. Esta afirmación oculta una complicación: la definición misma de lo que caracteriza un buen sonido vocal (y en este caso, un buen sonido coral). Cuando escuchamos un coro y nos llevamos una buena impresión del mismo, es evidente que, más allá de las características interpretativas de las versiones ejecutadas, podemos reconocer un tipo de sonido particular que es el resultado de un trabajo técnico-vocal implícito (MILLER, 1996; GUMM, 2009). Dicho trabajo técnico ha de haber considerado aspectos tales como: que todos los cantantes respiren de manera eficiente y al unísono, que los coreutas articulan las mismas vocales, que su tracto vocal está libre, que la dicción general sea precisa, y que tanto el ataque como la emisión del sonido se perciban sin esfuerzo, lo que normalmente conocemos como emisión y ataque normales o blandos (MAULEÓN, 2008).

Existen directores corales y profesionales de la voz que no conocen en detalle cómo está compuesto el instrumento vocal, cuáles son sus características más importantes, cuáles son los procesos nerviosos, sensoriales y motrices implicados en el proceso fonatorio, y que no son capaces de observar y percibir las dificultades y sensaciones que sus coreutas poseen. Resulta frecuente presenciar metodologías de trabajo en las que los directores corales se limitan a aplicar modelos estandarizados de respiración, emisión e incluso del imaginario vocal. Lo peligroso de este marco didáctico cimentado en la imitación de patrones técnicos y estilísticos radica en que el mismo no tiene en cuenta la particularidad del instrumento de cada cantante. Este tipo de instrucción determina que los resultados obtenidos sean disímiles: lo que es eficiente para unos, no necesariamente lo será para otros (BUNCH-DAYME, 2009; WARD-STEINMAN, 2010).

Por último, diremos que en la práctica coral tradicional, la noción de trabajo vocal se encuentra basada en la realización de una batería de ejercicios durante los primeros 
quince minutos de cada ensayo. De este modo, la ejercitación y la evocación consciente de los recursos técnico-vocales sólo tiene lugar en un porcentaje de tiempo de ensayo muy bajo. Esto determina que la conceptualización e instrumentalización de dichos recursos sea prácticamente imposible.

Para lograr un sonido coral compacto, técnicamente bueno y estéticamente bello, es necesario que los directores corales posean la capacitación suficiente en el campo de la Técnica Vocal, y con ello no nos referimos al hecho de cantar bien, ni tampoco a la posibilidad de asistir a unos pocos cursos sobre la temática, o leer ciertos libros que hacen al uso de vocabulario específico. Un guitarrista conoce a su instrumento tanto como a él mismo, puede percibirlo con sus sentidos, puede describirlo sin observarlo, y en la medida de su entrenamiento y su nivel de habilidad adquirida, puede ejecutarlo con mayor o menor grado de experticia. A su vez un guitarrista formado puede percibir a través del gesto de otro guitarrista qué nivel de experticia posee y asimilar las sensaciones que el ejecutante está teniendo.

\section{Algunas conclusiones}

El recorrido que hemos propuesto a lo largo de este artículo permite la reflexión respecto de las cualidades necesarias para los directores corales para desempeñarse efectivamente en el campo de la Técnica Vocal. En primer lugar, el director no sólo debe cantar bien, sino que debe conocer con qué instrumento tratará, para poder mejorar el sonido coral (MILLER, 1996). Tener conocimiento de los procesos involucrados en el canto, permitirá realizar un diagnóstico preciso de las necesidades vocales de los coreutas para guiar a los mismos hacia niveles de ejecución más profesionales (MCKINNEY, 2005).

Brindarles conocimiento certero acerca de su instrumento los habilitará también para constituirse, a largo plazo, en cantantes independientes que puedan poseer cierto dominio de su instrumento a través de sus percepciones fisiológicas y acústicas.

En segundo lugar, los directores corales no deben limitar la evocación de los recursos técnico-vocales a los primeros minutos de cada ensayo, sino hacer uso de los 
ejercicios en tanto herramientas de construcción de competencias de ejecución cada vez más diferenciadas. Al igual que cualquier atleta necesita poner a punto sus músculos para realizar un deporte; antes de dar inicio a la ejecución cantada, el coreuta precisa una rutina de precalentamiento de su instrumento. Pero además, el director coral debe ser el garante del desarrollo vocal sostenido de los individuos que participan de su agrupación coral. Con esto queremos decir que se vuelve insoslayable el diseño de una estrategia de entrenamiento vocal a largo plazo que contemple todos los aspectos importantes de la ejecución cantada. A través de la proyección de este dispositivo pedagógico, al director le será posible establecer un plan de trabajo corporal-vocal que le permita subsanar las deficiencias encontradas en su coro y, a su vez, elegir un repertorio adecuado (uno en el que el nivel de dificultad vocal que las obras requieren se encuentre alineado con el nivel de experticia vocal de los coreutas).

Los cantantes corales requieren muy a menudo apoyo técnico para resolver correctamente pasajes, frases y notas particulares de una obra. Aquellos directores que no poseen una correcta formación en el campo de la Técnica Vocal no tienen más opción que pasar por alto estas demandas sin brindarles el tratamiento necesario, lo cual produce inseguridad y frustraciones en los cantantes. Sin dudas, esta situación reflejará posteriormente en el sonido coral resultante.

En síntesis, consideramos indispensable un diálogo interdisciplinario entre la pedagogía de la práctica coral y los conocimientos teórico-prácticos de la Técnica Vocal para un despliegue de la actividad optimizado y efectivo. El desafío que enfrentan los directores corales en el siglo XXI se encuentra vinculado a la posibilidad de aprehender e instrumentalizar el conocimiento disponible en pos del perfeccionamiento constante y la mejora planificada del sonido vocal. Las capacidades de diagnóstico y prescripción de estrategias adecuadas y situadas constituyen la condición necesaria para el ejercicio de la profesión y el punto arquimédico a partir del cual restructurar la actividad toda bajo en el marco de un paradigma sólidamente fundamentado. 


\section{BIBLIOGRAFÍA}

BUNCH-DAYME, M. (2009). Dynamics of the Singing Voice. New York: SpringerWien.

GUMM, A. (2009). Making More Sense of How to Sing. Galesville: Meredith Music Publications.

HEIZMANN, K. (2003). Vocal Warm-ups: 200 Exercises for Choral and Solo Singers. Mainz: Schott Music GmbH \& Co.

JOHNSON, M. (1987). The body in the mind. Chicago: University of Chicago Press.

MAULEÓN, C. (1998). La pedagogía del canto. Aportes desde la investigación multidisciplinaria. Orpehotron(4), 86-94.

MAULEÓN, C. (2001). ¿Es la voz un instrumento musical? La Plata: Apunte interno de la Cátedra de Técnica Vocal 1 - FBA, U.N.L.P.

MAULEÓN, C. (2008). Las bases psicológicas de la interpretación en el canto. s.l.: Tésis doctoral inédita.

MCKINNEY, J. (2005). The Diagnosis \& Correction of vocal Faults: a manual for teachers of singing \& for choir directors. Illinois: Waveland Press.

MILLER, R. (1986). The Structure of Singing. New York: Schirmer Books.

MILLER, R. (1996). On the Art of Singing. New York: Oxford University Press.

PARUSSEL, R. (1999). Querido maestro, querido alumno: La educación funcional del cantante - el Método Rabine. Buenos Aires: Editorial GCC.

RABINE, E. (2002). Educación funcional de la voz. Método Rabine. Buenos Aires: Centro de Trabajo Vocal.

ROTHENBERG, M. (1981). Acoustic interaction between the glottal source and the vocal tract. En e. K.N. Stevens \& M. Hirano, Vocal Fold Physiology (págs. 305-323). Tokyo: University of Tokyo Press. 
SATALOFF, R. (1992). The Human Voice. Scientific American, 108-115.

SUNDBERG, J. (1987). The Science of the Singing Voice. Illinois: Northern Illinois University Press.

VENNARD, W. (1949). Singing, the Mechanism and the Technic. New York: Carl Fischer.

WARD-STEINMAN, P. M. (2010). Becoming a choral music teacher. New York: Taylor \& Francis. 